\title{
Percepção do consumidor frente aos riscos associados aos alimentos, sua segurança e rastreabilidade
}

\section{Consumer perception of risks associated with food, safety and traceability}

\section{Autores | Authors}

\section{$\bowtie$ Juliana Cunha de ANDRADE}

Instituto de Tecnologia de Alimentos (ITAL) Centro de Pesquisa e Desenvolvimento de

Carnes (CTC)

Av. Brasil, 2880

CEP: $13070-178$

Campinas/SP - Brasil e-mail: juliana@ital.sp.gov.br

Rosires DELIZA

Embrapa Agroindústria de Alimentos (CTAA)

Laboratório de Análise Sensorial e Instrumental

Rio de Janeiro/RJ - Brasi e-mail: rosires.deliza@embrapa.br

Eunice Akemi YAMADA

Instituto de Tecnologia de Alimentos (ITAL) Centro de Pesquisa e Desenvolvimento de Carnes (CTC) Campinas/SP - Brasil e-mail: eyamada@ital.sp.gov.br

\section{Maria Teresa Esteves Lopes} GALVÃO

Universidade Estadual de Campinas (UNICAMP)

Faculdade de Engenharia de Alimentos (FEA)

Departamento de Tecnologia de Alimentos (DTA)

Campinas/SP - Brasil e-mail:mtelgalv@fea.unicamp.br

\section{Lynn J. FREWER}

Newcastle University School of Agriculture Food and Rural Development Newcastle upon Tyne/Northumberland - United Kingdom e-mail: lynn.frewer@newcastle.ac.uk

\section{Nelson José BERAQUET}

Instituto de Tecnologia de Alimentos (ITAL) Centro de Pesquisa e Desenvolvimento de Carnes (CTC) Campinas/SP - Brasil e-mail: beraquet@ital.sp.gov.br

Autor Correspondente / Corresponding Author 


\section{Summary}

An increase in consumer interest in food safety has been observed in the last few decades. Governments have been pressed to adopt measures that assure the safety of foods and, as a consequence, legislation related to the contamination of food by physical, chemical and microbiological contaminants is being implemented. Within this context it is relevant to investigate the perception of Brazilian consumers on such issues. The present work evaluated the criteria used by consumers from Rio de Janeiro and Campinas to buy foods, and their vision of the risks of food contamination. The perception of consumers on traceability and its importance in contributing to the safety of foods was also evaluated using the Focus Group methodology. Three sessions were carried out (two in Campinas and the third in Rio de Janeiro). Differences were found between the consumers from the two cities. The Campinas consumers were more concerned and interested in the matter than those from Rio de Janeiro, since they reported looking for more information on the food labels. Mention was made of the risks of food poisoning, botulism, and pesticides by respondents in both cities. The main products considered as showing health risks were meats and seafood, the latter particularly in Rio. Products considered safe by consumers included fruits (without pesticides), vegetables and dried products. All the consumer groups were concerned about food contamination by pesticides and heavy metals. In relation to microbiological contamination, the three groups highlighted the risks of bacteria, especially Salmonella. Traceability was considered relevant by the three groups, since it should contribute to a more efficient product recall if necessary. However, from the consumer perspective, it will also lead to a price increase, although increasing consumer confidence in the safety of the foods. Many of the consumer concerns reflected information published in magazines and newspapers, such as the use of hormones in chicken. Industries and retailers should communicate scientific information about safety topics, thus contributing to consumer education.

Key words: Consumer; Contaminants; Traceability; Focus group. 


\section{Introdução}

Nos últimos anos, o consumidor tem se mostrado cada vez mais preocupado com os alimentos que consome (COSTA et al., 2000), tanto no que se refere à qualidade do produto em si como também com questões voltadas à segurança dos alimentos. Por essa razão, questões, como práticas higiênicas adequadas, métodos de produção, uso de pesticidas e biotecnologia têm sido consideradas no momento da escolha de um produto (COSTA et al., 2000).

A preocupação com a segurança dos alimentos tem levado a inúmeras alterações no processo de produção dos mesmos. Como exemplos, que ilustram essa mudança, há a proibição do uso de antibióticos na produção de frango a partir de 2012 pela União Europeia (MARVIN et al., 2009) e o crescente interesse em tecnologias alternativas para redução de carga microbiana (COSTA et al., 2000). No entanto, os consumidores ainda têm restrições a algumas tecnologias, como, por exemplo, o uso da irradiação para a conservação de alimentos.

Normalmente, os contaminantes classificados como químicos e microbiológicos (FAO e WHO, 2006) são considerados os mais importantes em relação ao risco a segurança e inocuidade alimentar (TENT, 1999); em razão disso, os produtores de alimentos e as instituições reguladoras tentam restaurar a confiança dos consumidores por meio da aplicação de sistemas de rastreabilidade dos alimentos e ingredientes (VAN RIJSWIJK et al., 2008). Contudo, a rastreabilidade só aumentará a confiança dos consumidores se os mesmos estiverem conscientes e informados a respeito desse sistema, bem como da forma como é aplicado pelos agentes da cadeia alimentar (RÖHR et al., 2005). Nesse sentido, conhecer as percepções dos consumidores sobre o tema 'segurança dos alimentos' torna-se extremamente relevante, pois pode influenciar, junto com outras variáveis socioeconômicas e demográficas, a escolha do alimento a ser consumido e contribuir para a efetividade das legislações a serem implantadas.

O método qualitativo focus group possui grande utilidade em estudos exploratórios sobre o comportamento do consumidor. O focus group é definido como um exercício de dinâmica de grupo, no qual a condução das sessões e a interpretação dos resultados obtidos devem ser entendidas dentro do contexto da interação dos participantes (STEWART et al., 2006). Esse método permite aos participantes explicar motivações e razões para as suas atitudes, percepções e preferências, por meio de interação, formulação de ideias; permite, ainda, aos participantes, tirar conclusões e respostas que, usando-se outros métodos de pesquisa, não seriam obtidas (MINIM, 2006). O presente estudo teve como objetivo investigar as percepções dos consumidores de duas grandes cidades brasileiras na compra e no consumo de produtos alimentícios em relação aos riscos químicos e microbiológicos associados ao consumo de alimentos, bem como avaliar o nível de conhecimento e envolvimento desses consumidores frente aos temas 'segurança dos alimentos' e 'rastreabilidade'.

\section{Material e métodos}

Foram conduzidas três sessões exploratórias de focus group em duas grandes praças brasileiras - Campinas-SP e Rio de Janeiro-RJ -, sendo duas em Campinas e uma no Rio de Janeiro. Em cada praça, foram contactadas 20 pessoas para participar da sessão de focus group. O índice de desistência no Rio de Janeiro foi maior, inviabilizando a execução de uma segunda sessão.

Cerca de dez pessoas participaram de cada uma das sessões de discussões. Os consumidores foram recrutados a partir de uma lista de indicações de familiares e conhecidos fornecida pela equipe de trabalho, considerando-se a diversidade em relação a idade e sexo, e a presença de crianças no domicílio. Ao todo, foram entrevistadas 29 pessoas - Campinas ( $n=19)$ e Rio de Janeiro $(n=10)$-, sendo 14 homens e 15 mulheres com idades variadas, de 20 a 65 anos, com predomínio da faixa etária de 30 a 50 anos. Cerca de $60 \%$ dos entrevistados trabalhavam fora em período integral, $70 \%$ deles apresentavam renda acima da média da população brasileira ( $R \$ 1.200,00)$ e curso superior completo. Nenhum dos participantes da pesquisa atuava diretamente com alimentos ou tinha conhecimento prévio sobre segurança dos alimentos. Para a realização das discussões, seguiu-se o protocolo pré-determinado pelo Projeto Sigma Chain (SIGMACHAIN, 2009) (www. sigmachain.eu), previamente utilizado com consumidores dos países Holanda, Irlanda, Polônia e França. O roteiro estabelecia quatro etapas. Numa primeira etapa, ou sessão introdutória, procurou-se conhecer os fatores que influenciam a escolha do local de compra e a percepção sobre de quem é a responsabilidade pela segurança dos alimentos. Na segunda etapa, foi abordado o tema dos riscos químicos e microbiológicos associados à segurança dos alimentos. O tema da comunicação sobre segurança dos alimentos foi abordado na terceira etapa e, finalmente, o estudo foi encerrado discutindo-se o tema 'rastreabilidade' e sua importância. O Quadro 1 ilustra um resumo dos tópicos abordados na discussão.

As sessões de discussão tiveram duração de aproximadamente duas horas cada. Um mesmo moderador conduziu todas as discussões, sendo acompanhado por dois assistentes; observe-se que todas as sessões foram gravadas. Na análise dos resultados das sessões de focus group, de acordo com Bryman (2012), primeiramente se fez a transcrição dos dados e, posteriormente, a análise do conteúdo, buscando-se estabelecer as categorias que foram exemplificadas por 
Percepção do consumidor frente aos riscos associados aos alimentos, sua segurança e rastreabilidade ANDRADE, J. C. et al.

trechos das falas dos participantes dos grupos. Antes do início das discussões, foi solicitado aos participantes que preenchessem um pequeno questionário sobre seus hábitos alimentares.

\section{Resultados e discussão}

O perfil dos consumidores com relação aos hábitos de consumo e compra de produtos é apresentado na Tabela 1

Os participantes do estudo no Rio de Janeiro demonstraram não rejeitar nenhum tipo de carne. Já $16 \%$ da amostra populacional entrevistada em Campinas reportou não consumir carne de frango. $\mathrm{Na}$ amostra pesquisada, também não foram encontrados consumidores vegetarianos.

Quadro 1. Tópicos abordados nas sessões de focus group.

1. Responsabilidade frente à segurança dos produtos adquiridos;

2. Percepção do consumidor ao comprar produtos em diferentes locais (supermercados, mercadinhos, fazenda, etc.);

3. Busca de informações sobre o produto na hora da compra;

4. Percepção de risco quando se fala em contaminação de alimentos;

5. Exemplos de produtos alimentares que o consumidor considera arriscados e seguros para consumo;

6. Contaminantes que o consumidor considera mais e menos perigosos;

7. Fonte de informações sobre produtos alimentares;

8. Rastreabilidade alimentar;

9. Vantagens e desvantagens da rastreabilidade da cadeia alimentar.
Mais da metade dos consumidores de Campinas (58\%) reportou consumir algum tipo de produto orgânico, enquanto que, para a amostra do Rio de Janeiro, o consumo desses itens foi bem inferior (20\%). Quando questionados sobre a compra de produto proveniente de fonte sustentável, cerca de $50 \%$ dos consumidores de Campinas responderam que, às vezes, compram. No Rio de Janeiro, observa-se um maior desconhecimento sobre esse tipo de produto/prática, já que 50\% dos consumidores mencionaram não saber o que significava a palavra 'sustentável'.

A relação dos consumidores com os alimentos normalmente consumidos é apresentada na Tabela 2.

Os consumidores de Campinas se mostraram mais interessados nos alimentos que consomem quando comparados aos do Rio de Janeiro, pois 100\% dos entrevistados daquela cidade demonstraram algum interesse pelos alimentos que consomem, sendo $68 \%$ deles, muito interessados. Já no Rio de Janeiro, apenas $20 \%$ apresentaram-se muito interessados e $10 \%$ mostraram desinteresse. Cerca de $70 \%$ dos consumidores de Campinas declaram buscar informações adicionais sobre os produtos consumidos com "regularidade" ou "sempre", enquanto que, no Rio de Janeiro, $60 \%$ reportaram que "raramente" ou "às vezes" buscam informações. Já a leitura de rótulos para obter informações nutricionais foi mais frequente, apesar de ainda ser mais baixa no Rio de Janeiro, em relação a Campinas. Nas duas praças estudadas, cerca de $40 \%$ dos entrevistados mencionaram buscar "regularmente" essas informações. A busca de outras informações no rótulo foi também mais baixa no Rio de Janeiro quando comparada com Campinas, cidade na qual $58 \%$ dos participantes deste estudo mencionaram que "regularmente" ou "sempre" leem o rótulo para buscar outras informações além da nutricional. Já na

Tabela 1. Hábitos de consumo e compra nas cidades de Campinas e Rio de Janeiro.

\begin{tabular}{|c|c|c|c|}
\hline \multirow{2}{*}{$\begin{array}{l}\text { Hábitos de } \\
\text { consumo }\end{array}$} & & \multicolumn{2}{|c|}{ Respostas (\%) } \\
\hline & & Campinas & Rio de Janeiro \\
\hline \multirow[t]{4}{*}{ Consumo de carne } & Consome todas as carnes & 79,0 & 100,0 \\
\hline & Consome todas as carnes, exceto frango & 16,0 & 0,0 \\
\hline & Vegetariano mas consome peixe & 5,0 & 0,0 \\
\hline & Vegetariano sem consumir peixe & 0,0 & 0,0 \\
\hline \multirow[t]{2}{*}{ Consumo de produto orgânico } & Sim & 58,0 & 20,0 \\
\hline & Não & 42,0 & 80,0 \\
\hline Hábitos de compra & Frequência & & \\
\hline \multirow[t]{6}{*}{ Compra de produto de fonte sustentável } & Nunca & 0,0 & 0,0 \\
\hline & Raramente & 11,0 & 10,0 \\
\hline & Às vezes & 53,0 & 20,0 \\
\hline & Regularmente & 21,0 & 20,0 \\
\hline & Sempre & 11,0 & 0,0 \\
\hline & Não sei o que é sustentável & 5,0 & 50,0 \\
\hline
\end{tabular}


Percepção do consumidor frente aos riscos associados aos alimentos, sua segurança e rastreabilidade ANDRADE, J. C. et al.

Tabela 2. Relação dos consumidores com os alimentos normalmente consumidos.

\begin{tabular}{|c|c|c|c|}
\hline \multirow{2}{*}{ Relação } & \multirow{2}{*}{ Frequência } & \multicolumn{2}{|c|}{ Respostas (\%) } \\
\hline & & Campinas & Rio de Janeiro \\
\hline \multirow[t]{5}{*}{ Grau de interesse por alimentos } & Muito interessado & 68,0 & 20,0 \\
\hline & Razoavelmente interessado & 32,0 & 60,0 \\
\hline & Neutro & 0,0 & 10,0 \\
\hline & Razoavelmente desinteressado & 0,0 & 10,0 \\
\hline & Muito desinteressado & 0,0 & 0,0 \\
\hline \multirow{5}{*}{$\begin{array}{l}\text { Busca de informações adicionais } \\
\text { sobre produtos consumidos } \\
\text { (com exceção do rótulo) }\end{array}$} & Nunca & 0,0 & 20,0 \\
\hline & Raramente & 16,0 & 30,0 \\
\hline & Às vezes & 16,0 & 30,0 \\
\hline & Regularmente & 37,0 & 10,0 \\
\hline & Sempre & 32,0 & 10,0 \\
\hline \multirow{5}{*}{$\begin{array}{l}\text { Leitura de rótulos para busca } \\
\text { de informações nutricionais }\end{array}$} & Nunca & 0,0 & 20,0 \\
\hline & Raramente & 5,0 & 30,0 \\
\hline & Às vezes & 16,0 & 10,0 \\
\hline & Regularmente & 42,0 & 40,0 \\
\hline & Sempre & 37,0 & 0,0 \\
\hline \multirow{5}{*}{$\begin{array}{l}\text { Leitura rótulos para obter } \\
\text { outras informações }\end{array}$} & Nunca & 0,0 & 10,0 \\
\hline & Raramente & 21,0 & 40,0 \\
\hline & Às vezes & 21,0 & 20,0 \\
\hline & Regularmente & 32,0 & 20,0 \\
\hline & Sempre & 26,0 & 0,0 \\
\hline
\end{tabular}

cidade do Rio de Janeiro, esse percentual decresceu para $20 \%$, enquanto que $50 \%$ mencionam que "nunca" ou "raramente" fazem isso.

Nas duas cidades, a escolha do local de compra dos produtos alimentícios é influenciada por vários fatores, como credibilidade do local, marca do produto, higiene e limpeza do estabelecimento, e rotatividade e categoria dos produtos, além das condições de armazenamento. No Rio de Janeiro, a localização (proximidade com o domicílio) também foi um fator considerado importante na hora de escolher o local de compra, como expresso nas seguintes frases:

"Eu me sinto melhor comprando em um supermercado melhor, grandes redes de supermercados é mais atento a isso [segurança dos produtos alimentícios]. No mercadinho, por exemplo, estou com medo, a carne fica degelando". (consumidor do Rio de Janeiro).

"Eu confio e desconfio do produto que está sendo exposto, e não do mercado". (consumidor de Campinas).

"Dependendo do local, tem outra rede do mesmo supermercado que eu acho que é bem melhor". (consumidor do Rio de Janeiro).

Carne e produtos cárneos foram citados como os itens alimentares que mais preocupam os consumidores no momento da compra. Condições de armazenamento e origem dos produtos foram fatores considerados imprescindíveis para determinar a compra. Para os consumidores de ambas as praças, as questões voltadas à segurança dos alimentos não é tarefa única do local de venda, mas também do fabricante e do consumidor, que manipula por último o alimento.

Quando questionados sobre a questão da confiança frente aos produtos comercializados diretamente da fazenda, em comparação com os comercializados em supermercados, observou-se que, para a maioria, a confiança está fortemente relacionada com o tipo de produto. Supermercados passam mais confiança, principalmente no que se refere aos perecíveis. Para outros itens, os consumidores sentem-se bastante seguros adquirindo de fazendas, como mostrado por alguns depoimentos abaixo:

\footnotetext{
"Um produto que não seja tão perecível, como o mel, poderia comprar tanto da fazenda como do supermercado, pois ele não oferece risco tão grande." (consumidor do Rio de Janeiro).
}

"Eu não sou radical nesse aspecto - o queijo da fazenda é mais gostoso que do supermercado." (consumidor de Campinas).

A maior preocupação dos consumidores, independentemente da praça estudada, foi relacionada com o prazo de validade dos produtos. A maioria comentou ser essa a primeira informação que buscam. Consumidores do Rio de Janeiro mencionaram também que consultam as informações nutricionais, principalmente sobre calorias. Preço também foi mencionado como um dos quesitos decisivos no processo de escolha: 


\begin{abstract}
"Primeiro prazo de validade. Isto é ponto passivo. Tem que olhar para o prazo de validade. E depois, vou ver o que tem dentro dele [produto]. Então eu olho esta parte do produto, da manufatura". (consumidor de Campinas).
\end{abstract}

"Calorias, gordura, além do preço, calorias é a primeira coisa". (consumidor do Rio de Janeiro).

Ao serem questionados sobre contaminação de alimentos, vários riscos foram espontaneamente levantados, assim como os principais produtos associados a esses. Os termos empregados são apresentados na Tabela 3.

Menções relacionadas a riscos, como intoxicação alimentar, botulismo e agrotóxicos foram comuns nas duas praças estudadas e, entre os principais produtos relacionados aos riscos apontados, ficaram as carnes de todos os tipos e os frutos do mar, como camarão, mariscos e ostras. Note-se que esses últimos foram muito enfatizados no Rio de Janeiro, provavelmente em virtude de esta ser uma cidade praiana. Enlatados e embutidos, além de maionese, também foram destacados.

Dentre os produtos considerados seguros pelos consumidores, salientaram-se as frutas (produzidas sem agrotóxicos), legumes e produtos secos/desidratados, como arroz, feijão, cereais, biscoito e chocolate. Ormenese et al. (2009), em seu estudo quantitativo sobre riscos e perigos dos alimentos na percepção dos consumidores, obtiveram resultados semelhantes aos coletados no presente estudo, pois produtos de pescados e derivados da carne bovina foram considerados significativamente de maior risco do que produtos à base de trigo e outros grãos, assim como a água mineral, as frutas e verduras, e os laticínios.

Os consumidores também relacionaram os principais contaminantes que consideram de risco para a saúde, tanto químicos como microbianos, os quais são apresentados na Tabela 4.

Quando se discutiu a contaminação de alimentos por compostos químicos, em todos os grupos observou-se grande preocupação com a contaminação por agrotóxicos e metais pesados. Além desses compostos, observou-se também preocupação com a questão dos conservantes utilizados nos alimentos processados.

Os consumidores de Campinas foram mais específicos do que os consumidores do Rio de Janeiro ao mencionarem os riscos microbiológicos, comentando sobre vírus, coliformes, cisticerco, além de Salmonella, que foi destacada em ambas as praças. Isso pode estar refletindo o fato de Campinas contar com centros de pesquisas importantes em alimentos e produção agrícola, favorecendo maior frequência de notícias veiculadas nos meios de comunicação local. Kleef et al. (2006), em estudo sobre a percepção de riscos associados ao consumo de alimentos em diferentes países, identificaram
Tabela 3. Riscos associados à contaminação de alimentos e produtos potenciais para causar danos.

\begin{tabular}{clc}
\hline \multirow{2}{*}{ Riscos Campinas } & Rio de Janeiro \\
& Intoxicação / contaminação & Intoxicação \\
& Botulismo & Botulismo \\
& Agrotóxicos & Agrotóxicos \\
& Hormônios & Mofo / fungos \\
& Antibióticos & Salmonella \\
& Conservantes & Coliformes \\
& Má conservação & Verminoses \\
Manuseio inadequado & \\
Produtos & Palmito & Carne suína \\
& Carnes & Frutos do mar \\
& Embutidos & Enlatados \\
& Enlatados & Embutidos \\
Defumados & Peixe \\
Patês & Maionese \\
Maionese & \\
\hline
\end{tabular}

Tabela 4. Contaminantes químicos e microbiológicos considerados de risco à saúde pelos consumidores.

\begin{tabular}{lcc} 
& Campinas & Rio de Janeiro \\
& Nitritos / nitratos & Agrotóxicos \\
& Metais pesados & Corantes \\
Contaminantes & Pesticidas & Acidulantes \\
químicos & Corantes & Hormônios \\
& Conservantes & Metais pesados \\
& Agrotóxicos & Água oxigenada \\
& Edulcorantes & \\
& Espessantes & \\
& Bactérias & Salmonella \\
& Salmonella & Fungos \\
Contaminantes & Vírus hepatite A & Bactérias \\
microbiológicos & Coliformes & \\
& Leptospirose & \\
& Cisticerco & \\
& Gripe aviária & \\
\hline
\end{tabular}

papel importante da mídia na percepção dos referidos riscos pelos consumidores, apesar de a mesma não ter grande impacto nas decisões de compra.

Dentre os riscos de contaminação por agentes químicos e microbiológicos, os consumidores de ambas as cidades citaram os contaminantes químicos como sendo os mais prejudiciais, pois seus efeitos demoram a surgir e são difíceis de diagnosticar. Essa percepção vai ao encontro do que preconiza Barendsz (1998): segundo o autor, embora a contaminação microbiológica seja percebida como a mais ameaçadora para a saúde humana, a presença de resíduos químicos também 
oferece uma grande ameaça, principalmente quando os efeitos são avaliados em longo prazo.

Quando questionados sobre os pontos das cadeias alimentares mais susceptíveis à contaminação, os consumidores de Campinas consideraram susceptível toda a cadeia, desde as áreas rurais até a estocagem no ponto de venda. Já os consumidores do Rio de Janeiro, apesar de considerarem também todas as etapas da cadeia, mencionaram de maneira mais enfática algumas etapas específicas, como no domicílio, no transporte ou na fábrica, como expressa a frase abaixo.

"Ração de frango, não sei o que colocam na ração de frango". (consumidor do Rio de Janeiro).

Resultados similares foram observados por Kleef et al. (2006), indicando a importância de sistemas de controle em toda a cadeia produtiva.

Quanto ao tema rastreabilidade e seu papel na segurança dos alimentos, os consumidores demonstraram interesse e conhecimento expressos pelas seguintes frases:

\footnotetext{
"Se você tem um produto rastreado você tem segurança alimentar - a segurança checa toda a cadeia, desde o começo." (consumidor de Campinas).
}

"Se existe rastreabilidade existe um controle sobre todas as etapas da cadeia". (consumidor do Rio de Janeiro).

Os consumidores de Campinas se mostraram mais interessados na disponibilização de informações sobre segurança dos alimentos, enquanto que os consumidores do Rio de Janeiro demonstraram desinteresse sobre o tema. Para estes últimos, devem existir profissionais e órgãos competentes para fiscalizar cada estágio da cadeia. Em ambas as praças estudadas, observou-se maior interesse no recebimento de informações de inocuidade de produtos cárneos e derivados. Em um estudo conduzido na Noruega, por Torjusen, Nyberg e Wandel, em 1999, citado por Sirieix et al. (2007), observou-se que $42 \%$ dos entrevistados também não demonstraram interesse em obter informações sobre os produtos consumidos.

O tema 'rastreabilidade' também foi relevante para todos os grupos, principalmente em momentos de crise, ou seja, se houver a necessidade de recolher produtos do mercado. Os consumidores ressaltaram a importância de conhecer o produto desde a origem para fornecer um caminho a ser rastreado, controlado e algumas medidas serem efetivadas. No entanto, sob a ótica do consumidor, a rastreabilidade irá encarecer os produtos, mas, com certeza, aumentará a confiança em relação à segurança dos alimentos, conforme mostram as menções abaixo.

"Com certeza aumenta a confiança porque mostra que tem um controle". (consumidor do Rio de Janeiro).
"Se a empresa tira do mercado, aumenta a confiança. Se é a fiscalização, reduz a confiança”. (consumidor de Campinas).

Resultados similares foram relatados por Van Rijswijk et al. (2008), indicando que a rastreabilidade contribuiu para elevar a confiança do consumidor, com o aumento da transparência da cadeia alimentar.

\section{Conclusão}

Com base nas entrevistas conduzidas, limitado no contexto do manuscrito, pode-se concluir que:

- Os consumidores demonstram grande preocupação com os riscos associados à contaminação, sejam estes químicos ou microbiológicos, com tendência para maior preocupação associada aos riscos químicos, pois os impactos somente podem ser diagnosticados em longo prazo e são menos perceptíveis;

- O conhecimento do consumidor sobre segurança dos alimentos e rastreabilidade sofre grande influência dos meios de comunicação. Isso é uma indicação de que caberia à indústria e aos estabelecimentos do varejo comunicar melhor sobre o tema, no sentido de desmistificar alguns pré-conceitos estabelecidos pelos consumidores;

- A preocupação e o envolvimento demonstrados com as questões de segurança e rastreabilidade sugerem que a indústria e os distribuidores de alimentos devem dar maior atenção a tais questões, a fim de atender às demandas do consumidor e, consequentemente, favorecer a satisfação com o produto.

\section{Agradecimentos}

Os autores agradecem ao Projeto Sigma Chain (contrato n. ${ }^{\circ}$ FP6 - 518451), pelo apoio financeiro da União Europeia.

\section{Referências}

BARENDSZ, A. W. Food safety and total quality management. Food Control, Kidlington, v. 9, n. 2-3, p. 163-170, 1998.

BRYMAN, A. Social Research Methods. 4th ed. Oxford: University Press, 2012. 840 p. PMid:22398310.

COSTA, M. C.; DELIZA, R.; ROSENTHAL, A.; HEDDERLEY, D.; FREWER, L. Non conventional technologies and impact on consumer behavior. Trends in Food Science \& Technology, London, v. 11, n. 4-5, p. 188-193, 2000. http://dx.doi.org/10.1016/ S0924-2244(00)00052-2

FOOD AND AGRICULTURAL ORGANIZATION OF UNITED NATIONS - FAO; WORLD HEALTH ORGANIZATION - WHO.

Food Safety Risk Analysis: A Guide for National Food Safety 
Authorities. Rome: WHO; FAO, 2006. (Food and Nutrition paper n. 87). Disponível em: <ftp://ftp.fao.org/docrep/fao/009/a0822e/ a0822e00.pdf>. Acesso em: 03 ago. 2009.

KLEEF, E. V.; FREWER, L. J.; CHRYSSOCHOIDIS, G. M.; HOUGHTON, J. R.; KORZEN-BOHR, S.; KRYSTALLIS, T.; LASSEN, J.; PFENNING, U.; ROWE, G. Perceptions of food risk management among key stakeholders: Results from a crossEuropean study. Appetite, London, v. 47, n. 1, p. 46-63, 2006. PMid:16584811. http://dx.doi.org/10.1016/j.appet.2006.02.002

MARVIN, H. J. P.; KLETER, G. A.; FREWER, L. J.; COPE, S.; WENTHOLT, M. T. A.; ROWE, G.A. Working procedure for identifying emerging food safety issues at an early stage: Implications for European and international risk management practices. Food Control, Kidlington, v. 20, n. 4, p. 345-356, 2009.

MINIM, V. P. R. Análise Sensorial: Estudo com Consumidores. Viçosa: Editora UFV, 2006. 225 p.

ORMENESE, R. C. S. C.; FARIA, E. V.; LEMOS, A. L. S. C.; MIYAGUSKU, L. Os riscos e perigos dos alimentos na percepção dos consumidores. Brazilian Journal of Food Technology, Campinas, v. II SSA, p. 49-56, 2009.
RÖHR, A.; LÜDDECKE, K.; DRUSCH, S.; MÜLLER, M. J.; ALVENSLEBEN, R. V. Food quality and safety-consumer perception and public health concern. Food Control, Kidlington, v. 16, n. 8, p. 649-655, 2005.

SIGMACHAIN. Database Contact Disclaimer Home Project Objectives Workplan. Disponível em: <http://www.sigmachain. eu>. Acesso em: 10 jul. 2009.

SIRIEIX, L.; GROLLEAU, G.; SCHAER, B. Consumers and food miles. In: AIEA2 AND SOBER INTERNATIONAL CONFERENCE, 2007, Londrina. Anais Eletrônicos... Londrina: UEL, 2007. Disponível em: <http://ideas.repec.org/p/umr/wpaper/200703. html>. Acesso em: 28 jul. 2009.

STEWART, D. W.; SHAMDASANI, P. N.; ROOK, D. W. Focus groups theory and practice. 2nd ed. London: Sage, 2006. $232 \mathrm{p}$.

TENT, H. Research on food safety in 21 st century. Food Control, Kidlington, v. 10, n. 4-5, p. 239-241, 1999.

VAN RIJSWIJK, W.; FREWER, L. J.; MENOZZI, D.; FAIOLI, G. Consumer perceptions of traceability: A cross-national comparison of the associated benefits. Food Quality and Preference, Barking, v. 19, n. 5, p. 452-464, 2008. 